\title{
Limitations of Orientation Dependent Microstructure Etching in Silicon
}

\author{
R. W. Ade and E. R. Fossum \\ Department of Electrical Engineering, Columbia University, New York, New York 10027
}

Anisotropic orientation dependent etching has been used extensively in the formation of silicon-based microstructures. The technique exploits differences between the etch rates of the low index crystallographic planes for some wet chemical etchants. In particular, solutions of $\mathrm{KOH}$ in water have been shown to etch $\{111\}$ planes up to several hundred times more slowly than $\{110\}$ and $\{100\}$ planes. This allows fabrication of precise three-dimensional structures in (110) and (100) oriented silicon wafers $(1,2)$. The resulting structures are typically cavities with sidewalls formed by the slow-etching $\{111 \mid$ planes. When the edge of a masking material is aligned with the trace of a 1111 plane (i.e., its intersection with the surface of the wafer), the etch proceeds with minimal undercutting and the shape of the cavity's opening remains sharply defined.

The trace of any plane is a line parallel to the cross product of the vectors normal to the plane and to the wafer. The angle of intersection between the plane and the wafer's surface is determined by the scalar product of the normal vectors. Thus, vector algebra can be used to find the proper mask orientation and to predict the geometry of the cavity. For example, the structure of (100) $\mathrm{Si}$ V-grooves (3) is based on the following:

(i) the planes (111) and(111) both intersect the (100) surface at an angle

$\Theta=\cos ^{-1}([100] \cdot[111] / \sqrt{ } 3)=\cos ^{-1}([100] \cdot[1 \overline{1} 1] / \sqrt{ } 3)=54.7^{\circ}$

(ii) the traces of these planes lie parallel to the direction

$$
[0 \overline{1} 1]=[100] \times[111]=-[100] \times[1 \overline{1} \overline{1}]
$$

which is typically indicated by a flat on the wafer perimeter. Thus, an orientation dependent etch (ODE) performed through a long rectangular mask opening oriented parallel to the flat yields a $\mathrm{V}$-shaped groove with a depth-to-width ratio equal to $\tan \left(54.7^{\circ}\right) / 2=\sqrt{ } 2 / 2$.

\section{Experiment}

In this note, we report on an attempt to employ the ODE technique for batch fabrication of cavities suitable for vertically coupling optical fibers to silicon circuitry. We have previously used laser-etched vertical cavities in photodetector structures designed to facilitate high density optical interconnection (4). As a processing alternative, orientation dependent etching of vertical shafts in (110) Si has been investigated. The cavity provides a mechanical seat for the optical fiber, and should consume a minimum amount of on-chip real estate. Cavities with cross sections approximating that of a single mode fiber core $(\sim 10 \mu \mathrm{m}$ in diameter) and depths greater than $20 \mu \mathrm{m}$ are suitable for this application. All sidewalls must be vertical, and adjacent walls must meet in a sharp edge (a [110] vector), where the transition between (111: planes does not cause deviations from verticality.

Previous demonstrations of microstructures etched perpendicular to (110) Si surfaces, such as sets of parallel trenches (5), usually involved one-dimensional mask geometries. In these cases, as in the above example of $(100)$ $\mathrm{Si} \mathrm{V}$-grooves, the crystallographic structure of the walls at the ends of the long mask openings may be ignored. When two-dimensional mask features were employed, e.g., to fabricate cavities for use as ink wells (6) or as microtool templates (7), verticality of every sidewall was not essential and was not reported. Our results indicate that it is possible to form cavities with two pairs of vertical walls, but that deep shafts composed exclusively of vertical walls cannot be obtained. This limitation is due to the existence of slow-etching $\{111\}$ planes which are not perpendicular to the (110) surface.

The experiments were conducted on boron-doped (110) Si with 10-12 $\Omega$-cm resistivity. The etchant was a 44 weight percent (w/o) solution of $\mathrm{KOH}$ in water, held at $85^{\circ} \mathrm{C}$. A $1 \mu \mathrm{m}$ thick layer of thermally grown $\mathrm{SiO}_{2}$ was patterned photolithographically to form a mask for the etch. The mask openings consisted of parallelograms with a variety of length-to-width ratios. Their sides were oriented parallel to the vectors [112] and [112], along the traces of the $(\overline{1} 11)$ and $(1 \overline{1} 1)$ planes. The $70.5^{\circ}$ angle between these vectors is matched by the acute angles of the

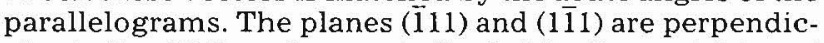
ular to the (110) surface, as indicated by the vanishing of the scalar products $[\overline{1} 11] \cdot[110]$ and $[1 \overline{1} 1] \cdot[110]$. The other (111) planes, (111) and (11) , intersect the surface ob-

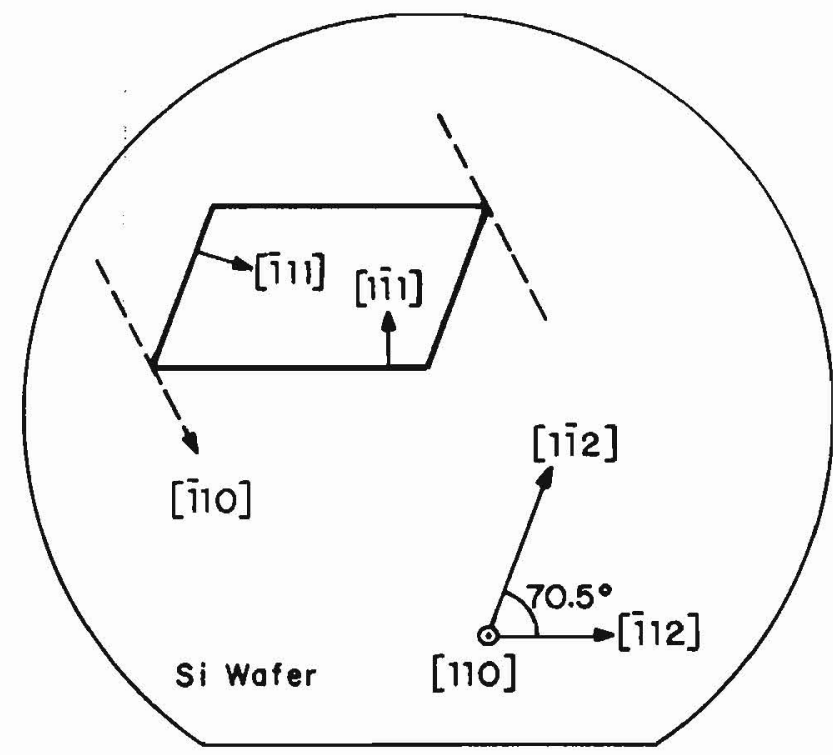

Fig. 1. Schematic diagram of the etch mask geometry, with relevant directions indicated. Dashed lines mark the traces of the planes (111) and $(11 \overline{1})$

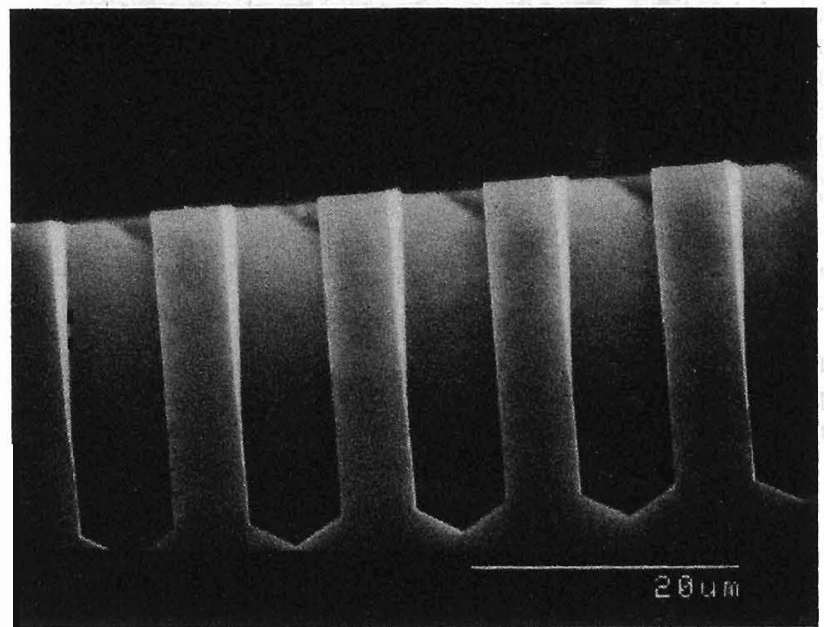

Fig. 2. Cross-sectional SEM photograph showing vertical-walled trenches etched in (110) Si. Etch time $=25 \mathrm{~min}$. 

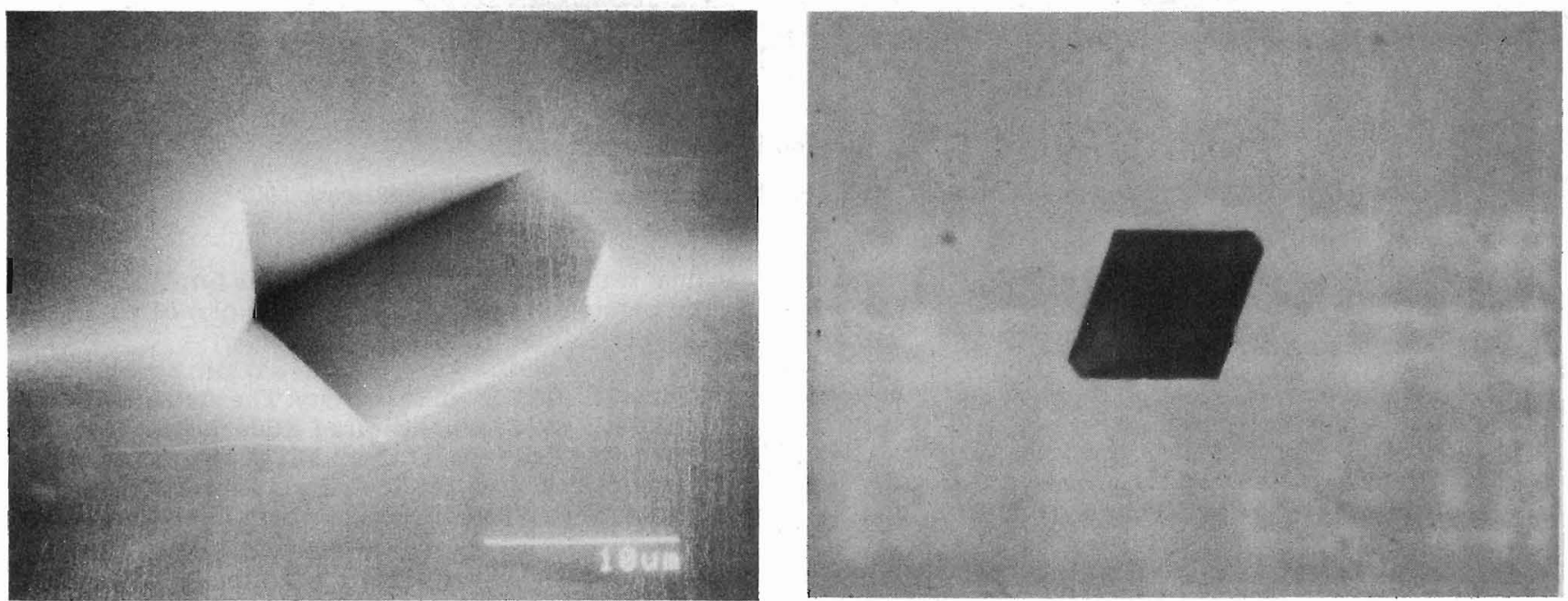

Fig. 3. (a, left) SEM photograph of a cavity etched in (110) Si through a rhomboidal mask opening with $16 \mu \mathrm{m}$ sides (b, right) top view of a typical etched cavity prior to cleaving.

liquely at an angle $\cos ^{-1}(2 / \sqrt{ } 6)=35.3^{\circ}$. A schematic diagram showing the mask geometry and several relevant crystallographic directions appears in Fig. 1.

A number of slits rotated successively by $3.0^{\circ}$ were also included in the photolithographic plates; they served to initially locate the desired traces and to set a maximum of $1.5^{\circ}$ on any alignment errors. Examination of etched trenches oriented parallel to the (111) and (11) traces confirmed the alignment accuracy, since they did not exhibit the jogs and ledges which result from misaligned etches (5). A cross-sectional scanning electron micrograph of one set of these trenches is shown in Fig. 2. Characterization of the ODE's was performed by means of optical and electron microscopy and a DEKTAK surface profiler. Etches ranging from $30 \mathrm{~s}$ to $50 \mathrm{~min}$ in duration were inspected, and etch rates of approximately 1 $\mu \mathrm{m} / \mathrm{min}$ were noted, in agreement with previously published work (2).

\section{Discussion}

The principal significance of the ODE experiments was their failure to attain structures with the desired geometry. This was particularly evident for parallelograms with length-to-width ratios approaching unity (i.e., for nearly rhomboidal openings in the $\mathrm{SiO}_{2}$ mask). The etched cavities were not parallelepipeds with four vertical walls, as predicted by a perpendicular projection of the mask geometry shown in Fig. 1. Instead, the cavities resembled those which would result from a slightly different mask geometry, in which the traces of the oblique \{111 planes (dashed lines in Fig. 1) also coincide with a straight segment of the mask edge. The cavities have six sidewalls rather than four, and the two additional walls intersect the surface at approximately $35^{\circ}$. When the mask opening is a rhombus, the oblique walls eventually meet in a line directly below the shorter diagonal of the rhombus, and the cavity depth is limited to one-half the length of the diagonal. Figure 3 shows a scanning electron micrograph of a cleaved cross section of a cavity etched for $10 \mathrm{~min}$, and a top view of an adjacent cavity, 3 min into the etch.

The plane (111) intersects the wafer's surface in one of the acute-angle corners of the parallelogram, and the plane $(11 \overline{1})$ is located in a symmetric position at the opposite corner. Due to the rounding of the corners of the $\mathrm{SiO}_{2}$ mask opening, the traces of these planes may actually be aligned to a microscopically straight section of the mask edge. However, this is not believed to play an essential role in the evolution of the cavity geometry. Instead, following accepted theories of crystallographic etching of semiconductors (8), we surmise that the oblique $[111 \mid$ planes would appear as cavity boundaries, even if no masking of their traces occurred (i.e., if the mask corners were ideally sharp). This result is due to the kinetics of the etching process and the manner in which a particular atomic plane may be "anchored." The latter is usually done intentionally with an etch-resistant mask, which protects some of the plane's atoms (those on the trace) from the solution. However, it is also possible for an unetched group of atoms on a densely packed 111\} plane to serve as an anchor for the entire plane. Because of this, even an unmasked sample of (110) or (100) silicon exhibits formation of $\{111\}$ bounded structures after exposure to an ODE. Our experiments show that the strong bonding between adjacent $\{111 \mid$ planes, which accounts for the ODE phenomenon, is also an important limitation on its useful application.

In order to obtain deep vertical-walled shafts using the technique, it would be necessary to create an overriding vertical anisotropy. For example, it may be possible to induce accelerated etching of the oblique!111! planes. An enhancement of the $\{111\} \mathrm{Si}$ etch rate in $\mathrm{KOH}$ has been observed under intense laser radiation, which causes localized melting and, hence, a disruption of crystallography (9). A laser beam propagating normal to the wafer's surface might, therefore, provide the necessary anisotropy, if its glancing incidence on the vertically oriented planes did not significantly increase their etch rates. Experiments designed to test this hypothesis have not been performed.

\section{Summary}

In summary, we have attempted to use orientation dependent etching to fabricate vertical cavities in (110) Si which have as sidewalls only the $|111|$ planes that are perpendicular to the surface of the wafer. Our results indicate that it is impossible to select such a limited subset of these planes (e.g., by masking their traces) and neglect the effect of the others. This approach succeeds in onedimensional trench etching applications because the effect of the oblique $\{111\}$ planes is seen only at the ends of the long trenches, which may be removed by cleaving. However, the two-dimensional shape of a small mask opening cannot be projected vertically into $(110) \mathrm{Si}$, because of the interference of the non-vertical $|111|$ planes.

\section{Acknowledgments}

This work was supported by the Defense Advanced Research Projects Agency under NOSC contact N6600185-C-0258.

Manuscript submitted March 9, 1987; revised manuscript received June 27, 1987.

Columbia University in New York assisted in meeting the publication costs of this article.

\section{REFERENCES}

1. E. Bassous, IEEE Trans. Electron Devices, ED-25, 1178 (1978).

2. K. E. Bean, ibid., ED-25, 1185 (1978). 
3. C. M. Schroeder, Bell Syst. Tech. J., 57, 91 (1978).

4. R. W. Ade, E. E. Harstead, A. H. Amirfazli, T. Cacouris, E. R. Fossum, P. R. Prucnal, and R. M. Osgood, Jr., IEEE Trans. Electron Devices, ED-34, 1283 (1987).

5. D. L. Kendall, Appl. Phys. Lett., 26, 195 (1975).

6. K. E. Petersen, IEEE Trans. Electron Devices, ED-26,
1918 (1979)

7. D. A. Kiewit, Rev. Sci. Instrum., 44, 1741 (1973).

8. H. C. Gatos and M. C. Lavine, Prog. Semicond., 9, (1965).

9. R. J. von Gutfeld and R. T. Hodgson, Appl. Phys. Lett., 40, 352 (1982). 